\title{
Pain Control and Functional Milestones in Total Knee Arthroplasty: Liposomal Bupivacaine versus Femoral Nerve Block
}

\author{
Stephen Yu MD $®$, Alessandra Szulc MA, Sharon Walton MD, \\ Joseph Bosco MD, Richard Iorio MD
}

Published online: 16 February 2016

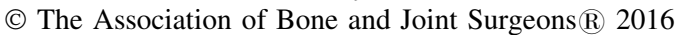

\begin{abstract}
Background Although pain management after total knee arthroplasty (TKA) affects rehabilitation, length of stay, and functional outcomes, pain management for patients undergoing TKA has yet to be standardized. Femoral nerve blocks (FNBs) are commonly used as an adjunct; however, these can result in transient quadriceps weakness and have been associated with in-hospital falls. Periarticular infiltration of liposomal bupivacaine has been recently introduced as a long-acting analgesic that can be administered without affecting motor function.

Questions/purposes (1) Does periarticular liposomal bupivacaine compared with FNB result in improved pain control as measured by pain scores and narcotic consumption? (2) How do liposomal bupivacaine and FNB compare in terms of gait and stairclimbing milestones and the proportion of patients who experienced a fall in the hospital?
\end{abstract}

All ICMJE Conflict of Interest Forms for authors and Clinical Orthopaedics and Related Research ${ }^{\circledR}$ editors and board members are on file with the publication and can be viewed on request.

Clinical Orthopaedics and Related Research ${ }^{\circledR}$ neither advocates nor endorses the use of any treatment, drug, or device. Readers are encouraged to always seek additional information, including FDAapproval status, of any drug or device prior to clinical use.

Each author certifies that his or her institution approved the human protocol for this investigation, that all investigations were conducted in conformity with ethical principles of research, and that informed consent for participation in the study was obtained.

One of the authors (RI) received a grant and personal fees from Pacira Pharmaceuticals (Parsippany, NJ, USA).

S. Yu ( $ه)$, A. Szulc, S. Walton, J. Bosco, R. Iorio

Hospital for Joint Diseases, NYU Langone Medical Center, 301

East 17th Street, New York, NY 10003, USA

e-mail: stephen.yu@nyumc.org; yus09@nyumc.org
Methods Between September 2013 and October 2014, a retrospective analysis was conducted involving 24 surgeons who performed a total of 1373 unilateral, primary TKAs. From September 2013 to April 2014, the routine approach to TKA pain management pathway consisted of preoperative administration of oral analgesics, intraoperative anesthesia (preferred spinal or general), an ultrasound-guided FNB, intraoperative analgesic cocktail injection, patient-controlled analgesia, and oral and IV narcotics for pain as needed. A total of 583 patients were included in this study group. Starting May 2014, FNBs were discouraged and there was department-wide adoption of liposomal bupivacaine. Liposomal bupivacaine became routinely used in all patients undergoing TKA with no other changes made to the multimodal analgesia protocol at that time, and 527 patients in this study group were compared with the FNB cohort. Chart review on a total of 1110 patients was conducted by a research assistant who was not participating in patient care. During the inpatient stay, pain scores during 8-hour intervals, narcotic use, and physical therapy milestones were compared. Results With the numbers available, we detected no clinically important difference in pain scores throughout the hospital stay; however, patients treated with liposomal bupivacaine consumed very slightly less narcotics overall (96 \pm 62 versus $84 \pm 73$ eq mg of morphine; [95\% confidence interval, 11-13 mg]; $\mathrm{p}=0.004)$ through postoperative Day 2 of inpatient hospitalization. Seventyseven percent (406 of 527) of patients receiving liposomal bupivacaine achieved their gait milestones of clearing 100 feet of ambulation versus 60\% (349 of 583) of patients receiving FNB $(\mathrm{p}<0.001)$ before discharge. Likewise, 94\% (497 of 527) of patients receiving liposomal bupivacaine completed stairs compared with $73 \%$ (427 of 583) of patients receiving FNB $(\mathrm{p}<0.001)$. Patients who received liposomal bupivacaine were less likely to experience a fall 
during the hospital stay than were patients treated with FNB (3 of 527 [0.6\%] versus 12 of 583 [2\%]; $p=0.03$ ).

Conclusions In the absence of strong data supporting FNB over liposomal bupivacaine, we have modified our TKA pain management protocols by adopting liposomal bupivacaine in lieu of FNBs, facilitating rapid rehabilitation while providing adequate pain control.

Level of Evidence Level III, therapeutic study.

\section{Introduction}

Perioperative pain management for patients undergoing TKA influences length of stay, rehabilitation progress, and functional outcome [9, 17, 20, 24]. Protocols vary widely across institutions; the general goals include improving analgesia and minimizing side effects and complications. The traditional approaches, pairing opioid patient-controlled analgesia with oral narcotics and the use of peripheral nerve blocks, have been shown to be effective in controlling pain [19]. Despite the effectiveness of narcotics, these agents are associated with side effects such as respiratory depression, hypotension, urinary retention, and postoperative ileus [11, 25]. Additionally, peripheral motor nerve blocks are associated with specific complications, including increased fall risk as a result of quadriceps weakness, potential injury to the nerve itself as well as increased resource utilization and total costs of care [10]. These adverse reactions can lead to increased hospital stays and delayed return to function and have prompted the search for alternative pain management strategies [5].

In May 2014, our institution adopted the use of intraoperative periarticular infiltration of liposomal bupivacaine. Liposomal bupivacaine has been recently introduced to the market as a long-acting analgesic agent using bupivacaine and a cytarabine liposomal delivery agent $[2,18]$. Liposomal bupivacaine has shown benefit in providing postoperative analgesic relief for up to 72 hours, specifically after bunionectomy [12] and TKA [4, 7], without affecting motor function. There is currently a lack of evidence that evaluates pain management protocols, comparing traditional pathways with modern pathways using liposomal bupivacaine, especially in large population studies. The ability to provide adequate pain control and decrease narcotic burden while avoiding motor blockade might facilitate rehabilitation and accelerate functional recovery.

We therefore asked: (1) Does periarticular liposomal bupivacaine compared with femoral nerve block (FNB) result in improved pain control as measured by pain scores and narcotic consumption? (2) How do liposomal bupivacaine and FNB compare in terms of achieving gait and stairclimbing milestones and the proportion of patients who experienced a fall in the hospital?

\section{Patients and Methods}

Institutional review board approval was obtained to perform a retrospective analysis at a single institution between September 2013 and October 2014. Twenty-four surgeons performed a total of 1373 primary TKAs. Patients were included for analysis if they underwent a unilateral, primary TKA. Patients were excluded from this study if they were receiving a simultaneous or second staged primary TKA, possessed inadequate records within the electronic medical record, or was a known deviation from standard protocol.

From September 2013 to April 2014, our routine approach to the TKA pain management pathway consisted of preoperative one-time administration of oral analgesics (200 mg celecoxib, $1000 \mathrm{mg}$ acetaminophen, $50 \mathrm{mg}$ pregabalin), intraoperative anesthesia (preferred spinal or general), an ultrasound-guided FNB (slow fractionated injection of $20 \mathrm{~mL}$ of $0.25 \%$ bupivacaine), intraoperative analgesic cocktail injection (40 cc $0.25 \%$ Marcaine [Hospira, Lake Forest, IL, USA], 5 cc of $5 \mathrm{mg}$ Duramorph [West-Ward, Eatontown, NJ, USA], and $1 \mathrm{cc}$ of $30 \mathrm{mg}$ Toradol [Regency, Shirley, NY, USA]), patient-controlled analgesia administered for the first 24 hours postoperatively (morphine, hydromorphone, or fentanyl), and oral and IV narcotics for maintenance and breakthrough pain as needed. A total of 719 patients were reviewed during this time period yielding 583 patients ultimately meeting inclusion/exclusion criteria.

Starting May 2014, department-wide adoption of liposomal bupivacaine began and became routinely used in all patients undergoing TKA at our institution. From May 2014 to October 2014, the standard of care and routine approach to the TKA pain management pathway were identical to the previous cohort with the exception of an intraoperative periarticular infiltration of liposomal bupivacaine and discontinued use of a FNB (Fig. 1). A total of 654 patients were reviewed during this time period yielding 527 patients ultimately meeting inclusion/exclusion criteria.

A total of 1110 patients were included in our study with approximately $19 \%$ of patients being excluded from each cohort as a result of deviations from the analgesia protocol and/or incomplete or missing data. All patients received identical departmental pre- and postoperative TKA protocols with the only difference in management being that 583 patients received a FNB and 527 patients received liposomal bupivacaine intraoperatively. Descriptive statistics of the two populations revealed no differences concerning 


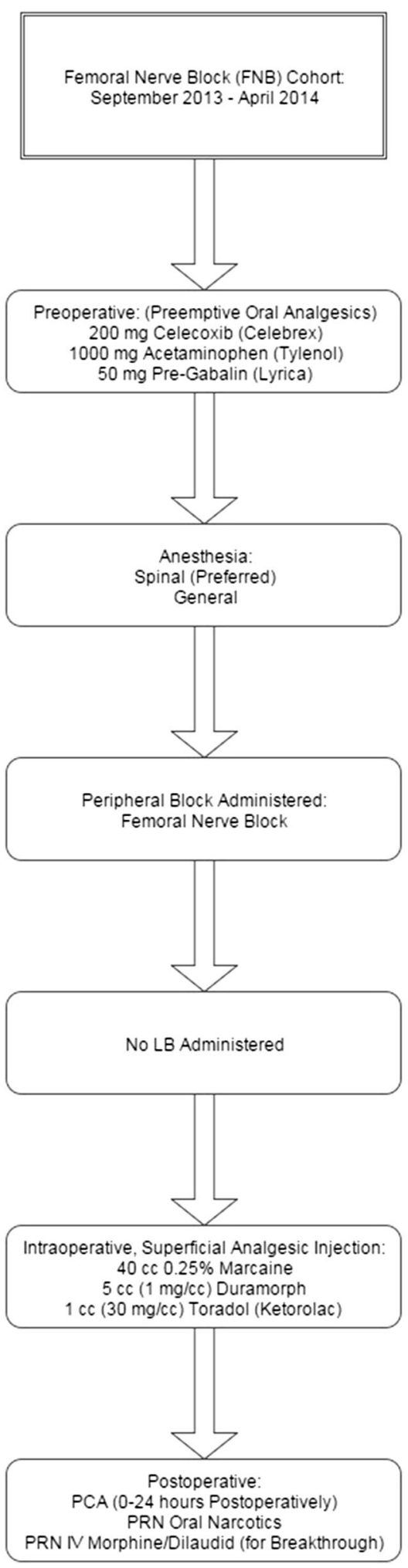

Fig. 1 This figure is a comparison between the pain management protocols between the femoral nerve block (FNB) and liposomal bupivacaine (LB) groups. The only difference between protocols is

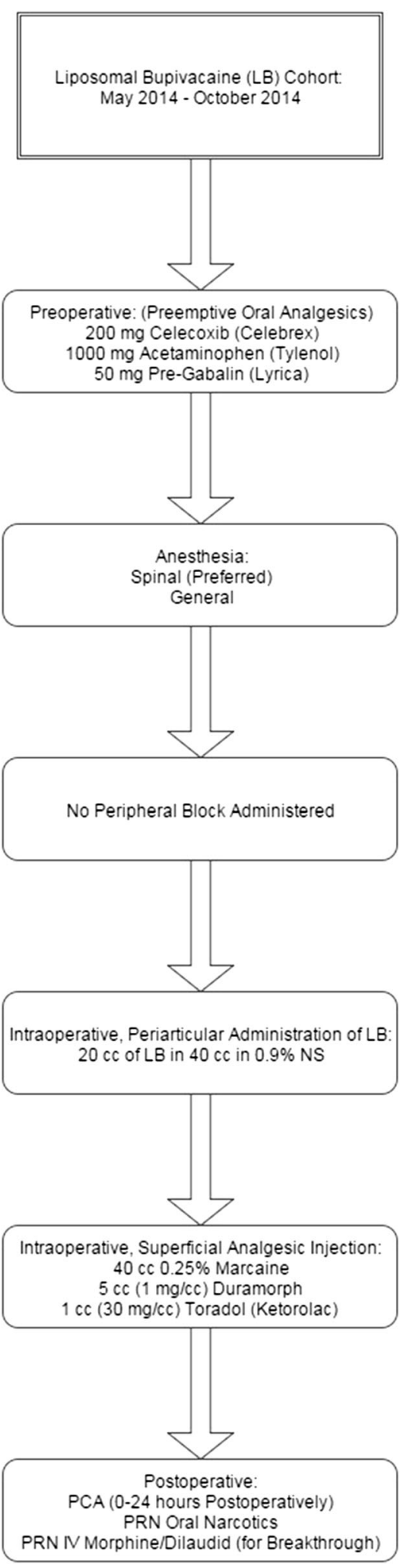

that the FNB group did not receive any LB (left) and the LB group did not receive a FNB (right). $\mathrm{PCA}=$ patient-controlled analgesia; $\mathrm{NS}=$ normal saline; PRN = as needed. 
Table 1. Descriptive statistics between the two cohorts*

\begin{tabular}{llllll}
\hline Cohort & Number & Age (years) $)^{\dagger}$ & Gender (female) & BMI $\left(\mathrm{kg} / \mathrm{m}^{2}\right)^{\dagger}$ & ${\text { Anesthesia }(\mathrm{spinal})^{\dagger}}^{\dagger}$ \\
\hline FNB, no LB & 583 & $66 \pm 10$ & $66 \%$ & $32 \pm 7$ & $93 \%$ \\
No FNB, LB & 531 & $65 \pm 11$ & $67 \%$ & $32 \pm 7$ & $91 \%$ \\
p value & & 0.588 & 0.751 & 0.461 & 0.137 \\
\hline ASA & FNB, no LB & Percent & No FNB, LB & Percent \\
\hline I & 13 & 2.26 & 13 & 364 \\
II & 400 & 69.57 & 150 & 68.81 \\
III & 161 & 28.00 & 2 & 28.36 \\
IV & 1 & 0.17 & 0.38 \\
\hline
\end{tabular}

The distribution across ASA groups between cohorts; $\mathrm{p}=0.869 ; *$ there were no significant differences among the two populations; ${ }^{\dagger}$ values are mean $\pm \mathrm{SD}$; ${ }^{*}$ anesthesia administered is expressed as percent spinal versus general; BMI = body mass index; FNB = femoral nerve block; LB = liposomal bupivacaine; ASA = American Society of Anesthesiologists.

age, gender, body mass index (BMI), American Society of Anesthesiologists (ASA) classification, and anesthesia type (Table 1).

\section{Liposomal Bupivacaine Administration}

All surgeons underwent standardized teaching protocols within the department and were instructed on correct liposomal bupivacaine technique. The liposomal bupivacaine injection (Exparel; Pacira Pharmaceuticals, Parsippany, NJ, USA) is prepared intraoperatively by the surgical technician before placing the final components in the knee. A homogeneous solution is created with $20 \mathrm{cc}$ of liposomal bupivacaine ( $13 \mathrm{mg} / \mathrm{cc}$ ) in $40 \mathrm{cc}$ of $0.9 \%$ normal saline solution. The total volume of $60 \mathrm{cc}(260 \mathrm{mg}$ of liposomal bupivacaine), once mixed, is placed into 3- to 20-cc syringes to be administered to the soft tissue surrounding the knee. The joint is adequately exposed and the analgesic is injected in a three-layer fashion with equal amounts injected into each of the (1) posterior capsule; (2) overlying periosteum, muscle, and fascia; and (3) the subcutaneous fat and subcuticular layer using a 21-gauge needle. Careful allocation of liposomal bupivacaine throughout the wound is essential for effectiveness, because liposomal bupivacaine does not diffuse to areas where it is not injected [14].

\section{Inpatient Hospitalization Period}

All patients received the standardized TKA clinical pathway and rehabilitation. Metrics recorded included (1) patient-reported pain scores, which were recorded by 8hour intervals, on the standard, 11 point Numeric Pain
Rating Scale; (2) narcotic administration, dosing, and patient-controlled analgesia use; and (3) physical therapy (PT) milestones, specifically when a patient was able to functionally achieve stairclimbing and ambulate greater than 100 feet. For patients who received a FNB, a knee immobilizer was used during the period of recovery to mitigate fall risk. The knee immobilizer was discontinued at the discretion of the physician and physical therapist when it was deemed that the motor blockade effects of the FNB had worn off. Postoperative inpatient falls are a departmental quality metric. Incidents are recorded and reported by nursing staff if a fall occurred.

\section{Data Abstraction}

Patient information was abstracted (SY, AS) from the electronic medical record (Epic Systems Corporation, Verona, WI, USA), including procedure type, date of surgery, age, gender, BMI, and ASA score. Pain scores were collected and recorded in 8-hour intervals starting from the time of postoperative floor admission to the time of discharge. Narcotic use was aggregated per postoperative day (POD) and converted to morphine equivalent dosages [15]. PT milestones (gait distance and stairclimbing) were recorded as the best effort for each POD.

\section{Statistical Analysis}

Descriptive statistics were used to compare the two cohorts of patients based on age, gender, BMI, and ASA score to compare the two populations. Standard independent-samples t-tests were used to detect statistical differences between the means of each outcome variable of the two 
groups and chi-square analyses were used for categorical data. A Mann-Whitney U statistical test was used to detect differences in median for narcotic use. Statistical significance was set at $\mathrm{p} \leq 0.05$.

\section{Results}

Small differences were detected in pain scores at three time points between the two groups; however, these differences were small enough that they are below the minimum clinically important difference (MCID) for a 10-point pain scale, which is approximately 2.0 points [21]. At 8 hours, the liposomal bupivacaine cohort reported less pain $(4 \pm 3$ versus $3.2 \pm 2.5[95 \%$ confidence interval $\{\mathrm{CI}\}, 0.13-$ $0.67], \mathrm{p}=0.012)$, but at the $64-(4 \pm 2$ versus $4 \pm 2$ [95\% CI, 0.02-0.58], $\mathrm{p}=0.049)$ and 72 -hour $(4 \pm 2$ versus $4 \pm 2$ [95\% CI, 0.12-0.68], $\mathrm{p}=0.018)$ time points, the FNB cohort reported less pain. No differences were observed at any other time points (hours 16-48, 80, and beyond; Fig. 2). Overall narcotic use was slightly decreased (96 \pm 62 versus $84 \pm 73$ eq $\mathrm{mg}$ of morphine [95\% CI, 11-13 mg]; $\mathrm{p}=0.004)$ for patients who received LB consuming $12 \pm 4$ equivalent $\mathrm{mg}$ of morphine less than patients who received a FNB during their length of stay. Patients receiving liposomal bupivacaine consumed slightly less narcotics during the first 48 hours of their postoperative admission (Fig. 3). After 48 to 72 hours (POD 2), narcotic consumption was not different between the two patient cohorts.

Patients receiving liposomal bupivacaine regained function faster and to a greater degree than patients receiving FNB. For gait, 406 of 527 (77\%) patients receiving liposomal bupivacaine achieved their gait

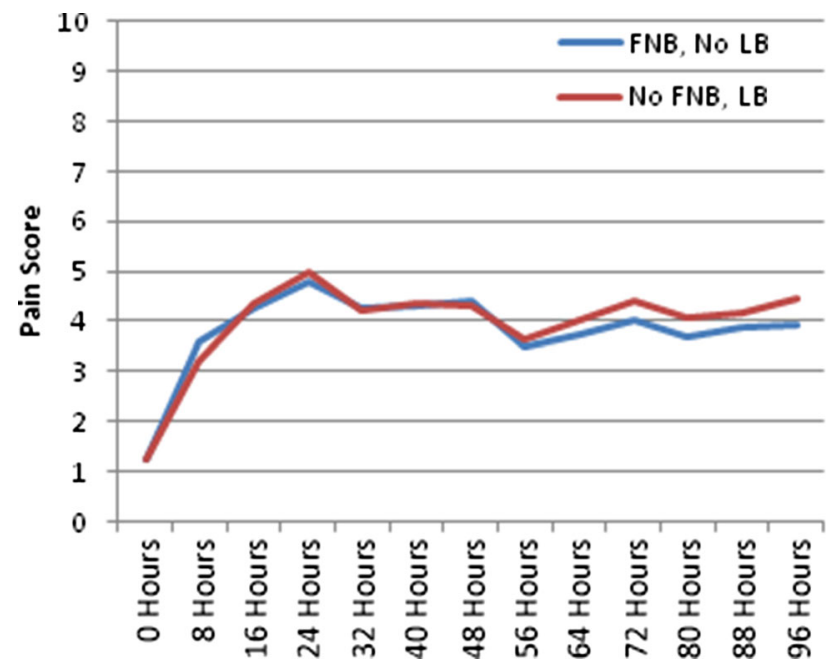

Fig. 2 Pain scores throughout hospital length of stay followed similar trends between both cohorts. FNB = femoral nerve block; LB $=$ liposomal bupivacaine. milestones (independent ambulation of 100 feet before hospital discharge) compared with 349 of $583(60 \%)$ patients receiving FNB (odds ratio [OR], 2.26 [liposomal bupivacaine exposure]; p < 0.001) (Fig. 4A). Regarding climbing stairs, 497 of 527 (94\%) patients receiving liposomal bupivacaine completed stairs during their inpatient hospitalization compared with 427 of $583(73 \%)$ patients receiving FNB (OR, 6.05 [liposomal bupivacaine exposure]; $\mathrm{p}<0.001$; Fig. 4B). Additionally, patients treated with liposomal bupivacaine had fewer inpatient falls than the FNB group (3 of 527 [0.6\%] versus 12 of 583 [2\%]; OR, 3.67 [FNB exposure], $\mathrm{p}=0.030$ ).

\section{Discussion}

Pain control after TKA is an important area of care that affects functional outcomes, length of stay, and patient satisfaction $[6,16]$. Pain management protocols are constantly evolving with varying multimodal strategies including regional anesthetic blocks as well as development of long-acting local anesthetics such as liposomal bupivacaine. Femoral nerve blocks are a common mode of adjunctive anesthesia for TKA and have been associated with delayed rehabilitation and fall risk [22]. To improve pain management protocols, minimization of the deleterious effects of the administered medications must be emphasized while maintaining adequate pain control. Our study demonstrated that patients receiving liposomal bupivacaine as an adjunctive mode of analgesia for TKA used slightly less narcotics, experienced comparable pain

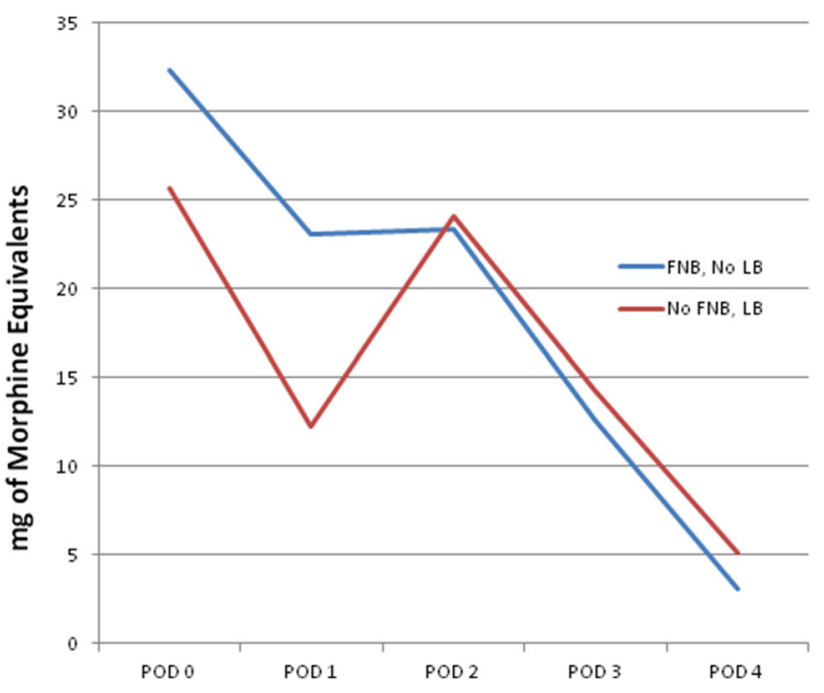

Fig. 3 The postoperative narcotic use throughout hospital length of stay demonstrated small decreases in narcotic use in the liposomal bupivacaine (LB) group, specifically during the first 48 to 72 hours postoperatively. FNB = femoral nerve block; POD = postoperative day. 

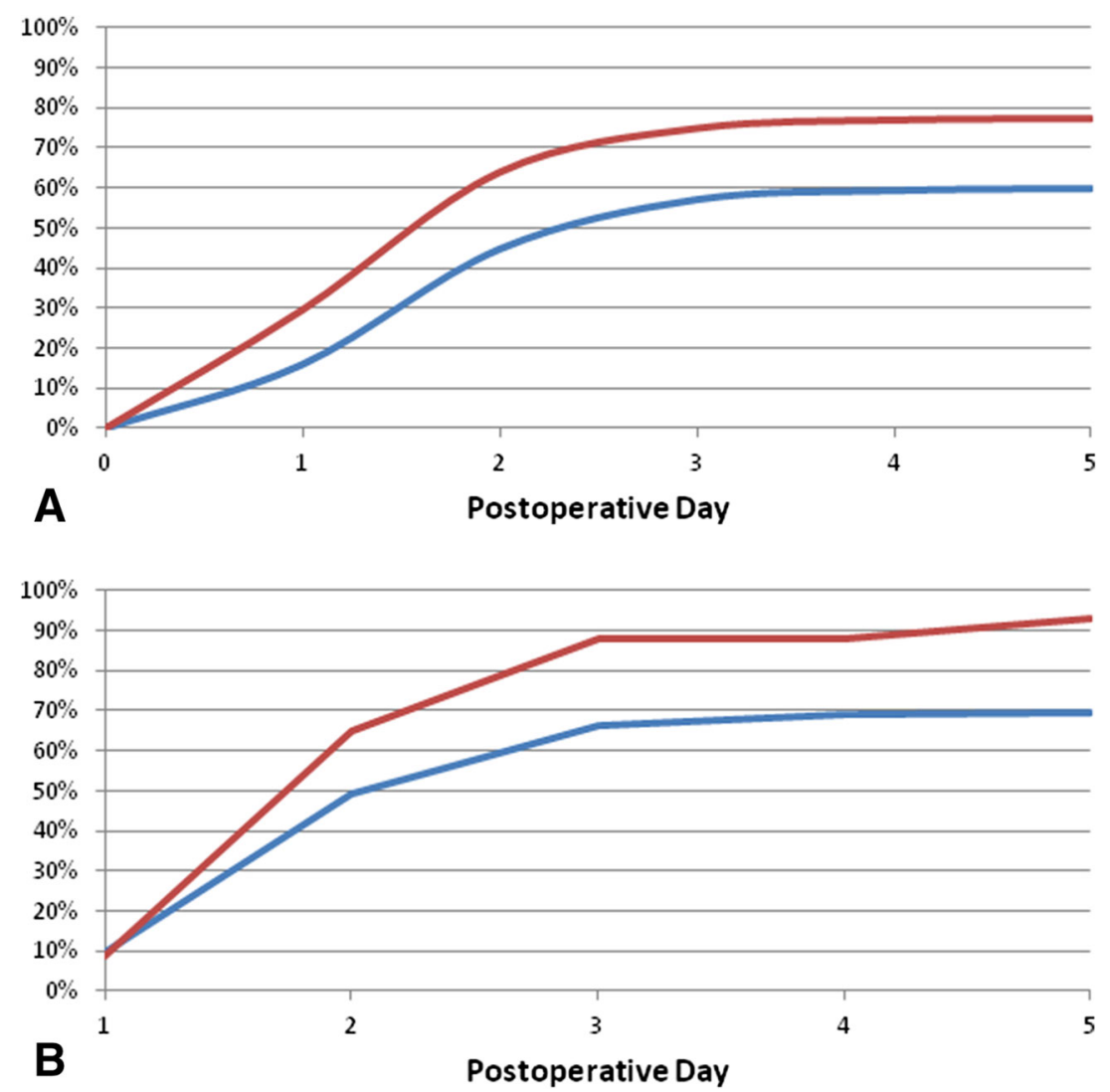

Fig. 4A-B Patients in the liposomal bupivacaine (LB) cohort completed their gait requirements of ambulating at least 100 feet to a faster and greater degree than patients receiving femoral nerve block

relief, and reached their functional milestones quicker compared with patients who received FNBs.

This study has several limitations. First, the data in this study were collected in a retrospective manner. Inherent to such a study design, lost or incorrect recording and retrieval of information may occur. Deviations from protocol may have not been identified in the electronic medical record. However, the large series of patients in both cohorts should minimize this error. A retrospective study is beneficial in regard to analyzing pain management protocols, because patient-reported pain scores can be quite subjective and possessing a large sample can alleviate the effects of varying perceptions of pain and potential selection biases of a prospective trial. Second, the study was historically controlled. This seems unlikely to represent a severe limitation, because the departmental protocols and TKA pathways were similar between the groups with only the previously described differences and because the time period over which both groups of patients were treated was short (approximately 1 year). Additionally, there may have been a learning curve with the introduction of liposomal bupivacaine, which could adversely influence its effect. The standard of care, departmental protocols, and even
(FNB) (A). Similarly, patients receiving LB completed stairs to a faster and greater degree than patients receiving FNB (B).

ancillary staff were scrutinized and were found to be consistent from admission to discharge with only one notable change: the transition from FNB to liposomal bupivacaine as an adjunctive pain control modality. Furthermore, the descriptive analysis of both time cohorts yielded no differences from patient demographics to type of anesthesia administered (Table 1). Third, attrition bias was not statistically corrected for in comparing the two cohorts. Although attrition may influence the results of the statistical analysis, it would intuitively only have effects after POD 2, at the point where most patients are discharged, which is beyond the scope of this study.

Lastly, another limitation was the number of surgeons included in the study. Although this study was conducted from a single institution and each surgeon went through standardized training for proper liposomal bupivacaine administration and technique, 24 surgeons have contributed to the database and there may have been variation in how liposomal bupivacaine was administered. The efficacy of liposomal bupivacaine is highly technique-dependent and although all surgeons were trained identically, there is no guarantee that the liposomal bupivacaine was administered with the same allocation technique in all wounds. Because 
liposomal bupivacaine has much less diffusion potential $(30 \%$ less) than standard bupivacaine and other injectable pain cocktail modalities, careful infiltrative technique is necessary for liposomal bupivacaine's analgesic effect to be realized. Likewise, preoperative administration of a FNB is also dependent on proper technique; however, the placement is localized and confirmed with ultrasound.

We found no clinically important differences in measured pain levels between cohorts. Barrington et al. [4] echoed similar results in a case-control study comparing 1000 joint replacements (including both hips and knees) using liposomal bupivacaine as an adjunct to the standard pain management regimen with a cohort of a 1000 previous cases receiving the standard pain management regimen only. Overall, they had slightly improved mean pain scores and a higher percentage of visual analog scale scores that were 0 , and likewise did not reach the MCID. However, our study demonstrated comparably effective results between liposomal bupivacaine and FNB, focusing on TKA populations only.

A slight and perhaps non-clinically important difference in narcotic consumption favoring the liposomal bupivacaine group was observed. Additionally, the observed decrease in narcotic consumption occurred during the first 2 days postoperatively, which could be explained by the reported window for clinical efficacy and pharmacokinetics reported for liposomal bupivacaine [13]. After approximately 72 hours, when the liposomal bupivacaine effect has worn off, the narcotic consumption in the two groups of patients was not statistically different. Dasta et al. [8] demonstrated findings favoring liposomal bupivacaine compared with patient-controlled analgesia in a combined gastrointestinal and orthopaedic surgery trial, in which they reported reductions in pain intensity scores, as measured by a pooled area under the curve analysis, and reductions in total opioid use over 72 hours postoperatively. The clinically related significance of the reduction in pain scores remains unclear; however, they found a reduction in opioid-related adverse events in patients receiving liposomal bupivacaine. Conversely, Bagsby et al. [3], in a small retrospective trial comparing liposomal bupivacaine with a traditional periarticular injection for TKA, did not find differences in total narcotic use and furthermore reported worse overall pain scores with liposomal bupivacaine. In the absence of strong findings favoring FNB over liposomal bupivacaine, our data suggest that liposomal bupivacaine poses an advantage over FNB with the ability to provide equivalent pain control, decreased narcotic use, and elimination of an additional procedure, which can cause nerve injury, quadriceps-induced weakness, and in-hospital falls.

Patients in the liposomal bupivacaine cohort achieved their PT milestones earlier and to a greater degree when compared with the cohort treated with FNBs. Surdam et al. [23], in a randomized trial comparing FNB with liposomal bupivacaine, demonstrated no difference in total and mean overall ambulation distance after TKA. They did however report that patients receiving liposomal bupivacaine had a higher mean ambulation distance on POD 1 as well as a greater proportion ambulating on the day of surgery. Our study compared milestone achievement as a functional parameter and the findings favored liposomal bupivacaine over FNB with respect to these milestones. Furthermore, although FNBs have been shown to effectively reduce pain after TKA [1], they have been associated with quadriceps weakness and in-hospital fall risk [22]. We found that patients treated with liposomal bupivacaine experienced fewer in-hospital falls than those who received FNBs.

We believe that liposomal bupivacaine is an attractive alternative to FNB in the management of postoperative pain after TKA, demonstrating slight decreases in overall narcotic use and no clinically important differences in pain control. As a result of eliminating FNBs, patients had improved functional parameters with earlier mobilization and greater achievement in PT as well as a decrease in proportion of inpatient falls. In the absence of strong data supporting FNB over liposomal bupivacaine, we have modified our TKA pain management protocol by adopting liposomal bupivacaine in lieu of FNBs. We believe that a large, randomized trial would be an important next step. Particular attention should be paid to comparing periarticular injections with liposomal bupivacaine alone, shortacting local anesthetics alone, and a combination of liposomal bupivacaine and short-acting anesthetics. This would provide a more definitive answer about the role of liposomal bupivacaine in perioperative total joint arthroplasty pain control.

Acknowledgments We thank and acknowledge Claudia Pulgarin and the DataCore team from the Department of Population Health for their assistance with this project.

\section{References}

1. Allen HW, Liu SS, Ware PD, Nairn CS, Owens BD. Peripheral nerve blocks improve analgesia after total knee replacement surgery. Anesth Analg. 1998;87:93-97.

2. Angst MS, Drover DR. Pharmacology of drugs formulated with DepoFoam $^{\mathrm{TM}}$. Clin Pharmacokinet. 2006;45:1153-1176.

3. Bagsby DT, Ireland PH, Meneghini RM. Liposomal bupivacaine versus traditional periarticular injection for pain control after total knee arthroplasty. J Arthroplasty. 2014;29:1687-1690.

4. Barrington JW, Dalury DF, Emerson RH Jr, Hawkins RJ, Joshi GP, Stulberg BN. Improving patient outcomes through advanced pain management techniques in total hip and knee arthroplasty. Am J Orthop. 2013;42(Suppl):S1-S20.

5. Benyamin R, Trescot AM, Datta S, Buenaventura R, Adlaka R, Sehgal N, Glaser SE, Vallejo R. Opioid complications and side effects. Pain Physician. 2008;11(Suppl):S105-120. 
6. Berend ME, Berend KE, Lombardi AV. Advances in pain management game changers in knee arthroplasty. Bone Joint J. 2014; 96(Suppl A):7-9.

7. Broome CB, Burnikel B. Novel strategies to improve early outcomes following total knee arthroplasty: a case control study of intra articular injection versus femoral nerve block. Int Orthop. 2014;38:2087-2089.

8. Dasta J, Ramamoorthy S, Patou G, Sinatra R. Bupivacaine liposome injectable suspension compared with bupivacaine $\mathrm{HCl}$ for the reduction of opioid burden in the postsurgical setting. Curr Med Res Opin. 2012;28:1609-1615.

9. Duellman TJ, Gaffigan C, Milbrandt JC, Allan DG. Multi-modal, pre-emptive analgesia decreases the length of hospital stay following total joint arthroplasty. Orthopedics. 2009;32:167.

10. Feibel RJ, Dervin GF, Kim PR, Beaule PE. Major complications associated with femoral nerve catheters for knee arthroplasty: a word of caution. J Arthroplasty. 2009;24:132-137.

11. Frater RA, Moores MA, Parry P, Hanning CD. Analgesia-induced respiratory depression: comparison of meptazinol and morphine in the postoperative period. Br J Anaesth. 1989;63:260-265.

12. Golf M, Daniels SE, Onel E. A phase 3, randomized placebo-controlled trial of DepoFoam ${ }^{\mathbb{R}}$ bupivacaine (extended-release bupivacaine local analgesic) in bunionectomy. Adv Ther. 2011;28:776-788.

13. Hu DD, Onel E, Singla N, Kramer WG, Hadzic A. Pharmacokinetic profile of liposome bupivacaine injection following a single administration at the surgical site. Clin Drug Invest. 2013;33:109-115.

14. Joshi GP, Cushner FD, Barrington JW, Lombardi AV Jr, Long WJ, Springer BD, Stulberg BN. Techniques for periarticular infiltration with liposomal bupivacaine for the management of pain after hip and knee arthroplasty: a consensus recommendation. J Surg Orthop Adv. 2015;24:27-35.

15. Knotkova H, Fine PG, Portenoy RK. Opioid rotation: the science and the limitations of the equianalgesic dose table. J Pain Symptom Manage. 2009;38:426-439.
16. Maheshwari AV, Blum YC, Shekhar L, Ranawat AS, Ranawat CS. Multimodal pain management after total hip and knee arthroplasty at the Ranawat Orthopaedic Center. Clin Orthop Relat Res. 2009;467:1418-1423.

17. Mullaji A, Kanna R, Shetty GM, Chavda V, Singh DP. Efficacy of periarticular injection of bupivacaine, fentanyl, and methylprednisolone in total knee arthroplasty: a prospective, randomized trial. J Arthroplasty. 2010;25:851-857.

18. Murphy TP, Byrne DP, Curtin P, Baker JF, Mulhall KJ. Can a periarticular levobupivacaine injection reduce postoperative opiate consumption during primary hip arthroplasty? Clin Orthop Surg. 2012;470:1151-1157.

19. Parvizi J, Miller AG, Gandhi K. Multimodal pain management after total joint arthroplasty. J Bone Joint Surg Am. 2011;93:1075-1084.

20. Ranawat CS, Ranawat AS, Mehta A. Total knee arthroplasty rehabilitation protocol: what makes the difference? J Arthroplasty. 2003;18(Suppl 1):27-30.

21. Salaffi F, Stancati A, Silvestri CA, Ciapetti A, Grassi W. Minimal clinically important changes in chronic musculoskeletal pain intensity measured on a numerical rating scale. Eur J Pain. 2004;8:283-291.

22. Sharma S, Iorio R, Specht LM, Davies-Lepie S, Healy WL. Complications of femoral nerve block for total knee arthroplasty. Clin Orthop Relat Res. 2010;468:135-140.

23. Surdam JW, Licini DJ, Baynes NT, Arce BR. The use of exparel (liposomal bupivacaine) to manage postoperative pain in unilateral total knee arthroplasty patients. J Arthroplasty. 2015; 30:325-329.

24. Tripuraneni KR, Woolson ST, Giori NJ. Local infiltration analgesia in TKA patients reduces length of stay and postoperative pain scores. Orthopedics. 2011;34:173.

25. Wheeler M, Oderda GM, Ashburn MA, Lipman AG. Adverse events associated with postoperative opioid analgesia: a systematic review. J Pain. 2002;3:159-180. 